\title{
Design and implementation of real time wideband channel simulator
}

\author{
Muhammad I Akram* and Asrar U Sheikh
}

\begin{abstract}
This paper describes the design and implementation of a DSP based real time wideband channel simulator. The simulator implementation uses a floating point (TMS320C6713) and a fixed point (TMS320C6416) DSPs.The simulator has 8 taps and baseband bandwidth of $20 \mathrm{MHz}$. It has flexibility to generate several channel models under varied environmental conditions. To validate the functionality of the simulator, the baseband data is applied to the simulator input and its output is statistically analyzed and the results are compared with those predicted analytically.
\end{abstract}

Keywords: Baseband, Channel models, Fixed point, Floating point, Simulator

\section{Introduction}

Wireless communications are inherently unreliable due to their time varying nature, multipath propagations and presence of interference signals from other users. In the presence of these impairments, substantially higher power must be transmitted to overcome these impairments inorder to acheive acceptable symbol error rate in any kind of radio channel. To design reliable and efficient wireless systems, it is essential to understand the behavior of radio channels in different environments.

Statistical channel modeling plays a vital role in the design of reliable wireless communication system. Channel modeling is the first step towards the efficient wireless system design. The purpose of channel modeling is to compute and estimate the various first and higher order statistical parameters of the fading channel. These parameters include Doppler spread, the time constants of fading, average fade duration, level crossing rates, amplitude probability distribution functions and the coherence bandwidth. For this purpose, measurements have been taken in different environment to characterize the channel.

Over the past few decades, a number of experiments have been performed to characterize mobile channels in urban, suburban, mountainous, wooded and highway environments [1-16]. On the basis of these measurements,

*Correspondence: miakram@kfupm.edu.sa

Department of Electrical Engineering, King Fahd University of Petroleum \& Minerals, Dhahran, 31261 Saudi Arabia several channel models have been proposed to explain the observed statistical nature of the fading channels between fixed base stations and mobile stations. These include short term fading models like the well-known Rayleigh, Rice [17], Hoyt [18], Nakagami-m [19] and Weibull [20] and for longer term lognormal model has been used $[6,21]$.

To evaluate the design and performance of a communication system, it is desirable to evaluate it in realistic situations. The experiments can be performed directly in a vehicle, driving through different environments. However, this is a time-consuming and expensive task and it requires the presence of measurement equipments with proper calibrations. Moreover, the field trials can be affected by unintended uncontrollable circumstances. The inexpensive and flexible option is to use a real time channel emulator and measure the performance in a laboratory environments as in [22-24]. The commercially available channel emulators available may not offer the user enough flexibility when configuring the wireless channel parameters to test the system under different environmental conditions. Such simulators do not cover V2V scenarios under different channel models like Hoyt, Rice Hoyt etc. A low cost channel simulator is therefore required that models different scenarios and at the same time provide the user flexibility to measure the performance of the wireless transceiver under environmental conditions.

Over the past few decades, efforts have resulted in several designs and implementation of real time simulators. Early efforts were based on analog components

\section{至 Springer}

(C) 2012 Akram and Sheikh; licensee Springer. This is an Open Access article distributed under the terms of the Creative Commons Attribution License (http://creativecommons.org/licenses/by/2.0), which permits unrestricted use, distribution, and reproduction in any medium, provided the original work is properly cited. 
[25-28]. The development of real time simulator starts in 1973 when [25] developed the first Rayleigh based channel simulator. The simulator used Zener diode to generate Gaussian random variable. But with the advent of digital computers, micro-controllers, fast Analog to Digital Converters (ADCs) and Digital to Analog Converters (DACs), the analog components were replaced by digital thereby increasing the reliability and flexibility of simulators. Comroe et el [29] first used discrete digital logic in its simulator. With the development of High Speed Digital Signal Processors (DSPs), the DSP based simulators were developed. Goubran et al [30] used 16 bit fixed point DSP for implementation and simulated the Gaussian quadrature components along with the log-normally distributed Line of Sight (LOS) component. Turkmani et al [31] used TMS320 E15 DSP chip for the development of a narrow-band simulator. Cullen et al [32] reported a frequency selective simulator using TMS32050 DSP and IMSA110 integrated circuits. It had a baseband bandwidth of $10 \mathrm{MHz}$ and maximum Doppler frequency of $100 \mathrm{~Hz}$. Chen et al [33] used TMS320C31 DSP to design a frequency selective simulator. Salkintzis et al [34] developed 6 taps wide-band channel simulator having maximum signal bandwidth of $20 \mathrm{MHz}$. It used two 32 bit DSP floating point processors. Papenfuss et al [35] used a hybrid DSP FPGA architecture to build a wide-band channel simulator. It was capable of simulating 12 delay taps and had a baseband bandwidth of $5 \mathrm{MHz}$. Satellite Channel simulator has been developed in [36] using TMS320C6701 DSP platform. Kominakis et al [37] developed a narrow-band fast and accurate simulator. Khars et al [38] developed a $5 \mathrm{MHz} 12$ taps wide-band simulator using 12 DSPs (1 for each tap) for the generation of complex coefficients. A narrow-band DSP based channel simulator has also been developed in [39]. Over the last decade, the use of Field Programmable Gate Arrays (FPGAs) in DSP applications has become quite common. The FPGA based simulators have also been developed and their implementations have been described in [40-45].

One important application of wireless communication is Vehicle to Vehicle (V2V) communications where both the transmitter and receiver are in motion. The V2V communication finds its applications in mobile ad-hoc wireless networks, intelligent highway systems, emergency, military and security vehicles. The implementation of V2V communications enhances road safety due to reduction in the number of accidents, improvement in highway traffic flow efficiency and real time data sharing without involving the cellular network which leads to efficient fuel consumption and reduced travel time. The statistical model for vehicle to vehicle communication was first proposed by Akki and Haber [46] and its statistical properties reported in [47]. This model covers Rayleigh distribution only with both inphase and quadrature components having identical variances. Matolak et al [48] after performing measurements in five different cities, models the channel as Weibull fading channel. Based on the work of [47], many V2V simulators have been designed and implemented. Cox et al [49] presented a discrete line spectrum based approach to simulate the channel. The work in [50] was based on sum of sinusoids (SOS) approach for simulator design. The simulator proposed in [51] is based on Kullback-Leibler divergence which is compared with IFFT based approach of simulator design. Borries et al [52] used Gaussian quadrature rules for simulator design. Zaji et al [53] proposed an efficient sum of sinusoids (SOS) based approach for V2V simulator design. All the simulator design approaches mentioned above are restricted to V2V Rayleigh fading channel only.

The simulators mentioned above may be classified into three categories. The first category uses the sum of sinusoid (SoS) approach for generating the fading channel coefficients. This approach has the drawback of multiple Sine function calls which makes it computationally expensive to implement in real time. Secondly this kind of simulator does not produce the channel with the statistical properties that match with the theoretical values. Another class of simulators uses IFFT based approach to generate the required channel coefficients. This approach is computationally efficient. The drawback is that it works on a block of data and can not be used for the streaming data in real time. The approach used in this paper, is the Filter based approach. This is computationally efficient as well as produces channel coefficients having more accurate statistical properties.

The proposed simulator is a modified form of the simulator described in [37]. The proposed simulator uses a generalized Wideband Nakagami Hoyt with diffused line of sight channel model for Vehicle to Vehicle communication [54] environment to generate real time fading data. It covers Rayleigh, Rice, Rice-Hoyt, Lognormal and static channels as its special cases. The multipaths have been modeled as Tap Delay Line (TDL) filter. Efficient implementation of optimal TDL filter has been performed over the TMS320C6416 DSP processor. The novel simulator implementation uses two DSP (TMS320C6713 and TMS320C6416) boards along with one wide bandwidth (Microline ORS114) I/O daughter board. To the best of authors' knowledge, to date such real time simulator has not been proposed and implemented.

The remainder of this paper is organized as follows. Section 2 presents the brief overview of the proposed Diffused Nakagami-Hoyt V2V channel model. Section 3 describes the channel simulator design philosophy and architecture. Section 4 shows the outcome of the simulator and their comparison with the analytical expressions. Finally, Section 5 concludes the paper. 


\section{Brief channel model description}

In V2V communications, the received signal consists of direct (LOS) and indirect (NLOS) component. The direct component may or may not be present depending on the presence or absence of obstacles between the transmitter and the receiver. The direct component may be further divided into a clear LOS between the receiver and the transmitter or a diffused LOS. The value of diffused LOS is negligible when the buildings are of steel or reinforced concrete but they must be considered for the wooden and bricks building. In rural areas, most of the building are made of wooden or bricks wall hence while modeling the channel, the diffused LOS component must be considered [55]. In V2V communications as mentioned by [56,57], when the antennas are inside the car, the shadowing must be considered due to the presence of roof top surface.

Youssef et al [16] established after taking the measurements in the rural environment that the channel is more accurately modeled only when the variances of Inphase and Quadrature components are different. The argument was further supported by [58] where the model matches the measured data for the cases of unequal variances. For V2V communication, [57] explained the case when the distance between the vehicles exceeds 70-100 m, the Nakagami $\mathrm{m}$-factor is observed to be less than unity, which corresponds to the case of unequal variances of the Gaussian quadrature components. Further as found from the $\mathrm{V} 2 \mathrm{~V}$ measurements (antennas inside car) results in $5 \mathrm{GHz}$ frequency band [48,59], the $m$ value of each tap of the channel model described is found to be less than unity (0.75-0.89) which from [16] corresponds to the value of $q$ (0.5-0.707).

Based on these, Akram et al [54] proposed Nakagami Hoyt V2V model with diffused line of sight under the assumption that omnidirectional antennas have been used. The proposed channel model $H(t)$ is a generalized model that covers the V2V environment as well.

$$
H(t)=\rho(t)+\mu(t)
$$

It considers, the lognormally distributed diffused LOS component $\rho(t)=A e^{z(t)}$ and a NLOS component having Hoyt distributed amplitude envelop $\mu(t)=\mu_{1}(t)+j \mu_{2}(t)$ under the assumptions that both transmitter and receiver are in motion. $\mu_{1}(t), \mu_{2}(t)$ and $z(t)$ are the real Gaussian random processes with zero mean and variances $\sigma_{1}^{2}, \sigma_{2}^{2}$ and $\sigma_{3}^{2}$ respectively and $A$ is the direct LOS component. $q=\frac{\sigma_{1}}{\sigma_{2}}$ and $V_{2}$ and $V_{1}$ are the velocities of transmitter and receiver respectively and $a=\frac{V_{2}}{V_{1}}$.

The time autocorrelation function of the random process $H(t)$ is derived in [54]

$$
\begin{aligned}
R_{H}(\Delta t) & =A^{2} \exp \left[\sigma_{3}^{2}\left(1+J_{0}\left(2 \pi f_{m 3} \Delta t\right)\right)\right] \\
& +\frac{1+q^{2}}{2 q^{2}} \sigma_{1}^{2} J_{0}\left(K V_{2} \Delta t\right) J_{0}\left(K V_{1} \Delta t\right)
\end{aligned}
$$

where, $J_{0}($.$) is the zero-order Bessel function. q=\frac{\sigma_{1}}{\sigma_{2}}$, $V_{2}$ and $V_{1}$ are the velocities of transmitter and receiver respectively, $K=\frac{2 \pi}{\lambda}$ and $f_{m 3}$ is the LOS component maximum Doppler.

The power spectral density of the proposed model is found as [54],

$$
\begin{aligned}
S_{H}(f) & =\int_{-\infty}^{\infty} R_{H}(\Delta t) e^{-j 2 \pi f \Delta t} d \Delta t \\
& =S_{\rho}(f)+S_{\mu}(f)
\end{aligned}
$$

where,

$$
S_{\rho}(f)=A^{2} e^{\sigma_{3}^{2}}\left[\delta(f)+\sigma_{3}^{2} f_{m 3} \frac{\operatorname{rect}\left(\pi f / f_{m 3}\right)}{\sqrt{f_{m 3}^{2}-4 \pi^{2} f^{2}}}\right]
$$

for $\sigma_{3}<0.3$ and,

$$
\begin{gathered}
\operatorname{rect}(t / T)=1, t \leq|T| \\
=0, \text { otherwise } \\
S_{\mu}(f)=\frac{1+q^{2}}{2 q^{2} \pi^{2} f_{m 1} \sqrt{a}} \sigma_{1}^{2} \\
\times K\left[\frac{(1+a)}{2 \sqrt{a}} \sqrt{1-\left(\frac{f}{(1+a) f_{m 1}}\right)^{2}}\right]
\end{gathered}
$$

where $K($.$) is the elliptical integral function of first kind,$ $a=\frac{V_{2}}{V_{1}}, f_{m 1}, f_{m 2}$ are the maximum Doppler shifts due to the motion of the receiver and transmitter respectively with $f_{m i}=\frac{V_{i}}{\lambda}$. Therefore, $f_{m 2}=a f_{m 1}$.

\section{Simulator description}

\subsection{Design philosophy}

In efficient real time systems design all the available system resources are efficiently utilized in order to minimize the cost and maximize the productivity. For the data acquisitioning at high data rate, the DSPs can not be interfaced directly with high speed ADCs and DACs

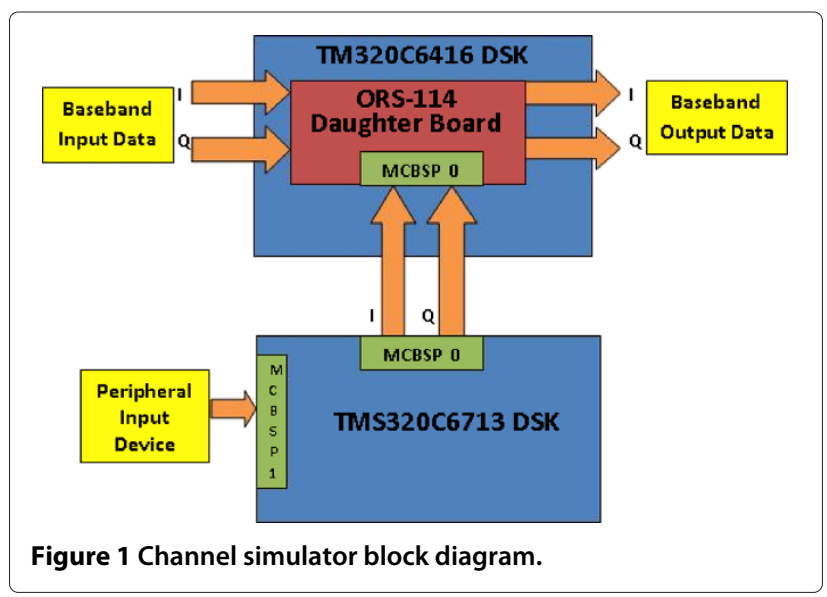




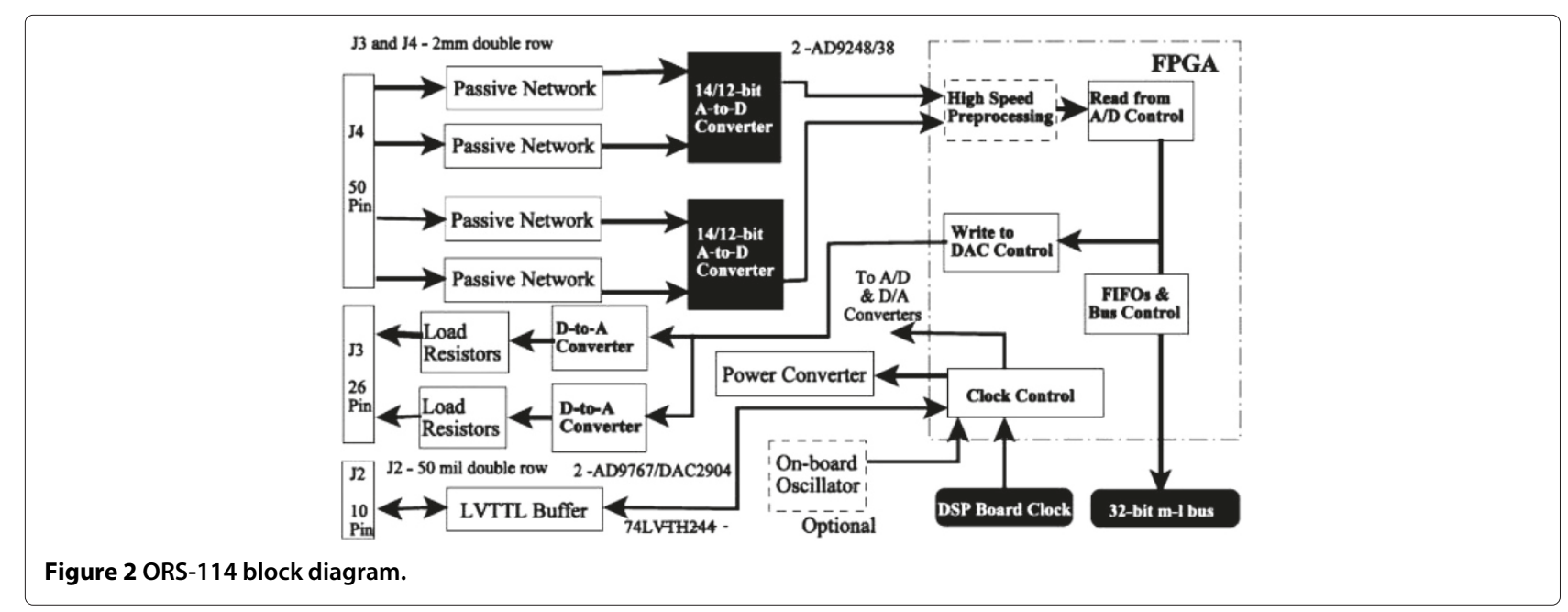

because of its I/O bandwidth limitations. The best solution is to use FPGA for this purpose. Therefore, Microline ORS114 daughter board was used for this purpose. The board consists of a vertex-2 FPGA, multiple channel ADC and DAC, FIFO memory and control circuitry used to synchronize the data input output events with DSP. The board is mounted over the peripheral expansion of TMS320C6416 fixed point DSP Starter Kit (DSK) which performs the TDL filtering. Since filtering operation needs to be performed at high data rate, for this purpose an optimal TDL filter need to be implemented. This can be done over fixed point processor of high clock rate. Hence for that purpose TMS320C6416 processor with $1 \mathrm{GHz}$ clock have been selected. The channel coefficient generation depends upon the time variations of the scatterers surrounding the transmitter and receiver. These variations are normally much slower as compared to the baseband data rate. Therefore for the channel generation purpose a processor with lower clock rate is adequate and a TMS320C6713 32 bit floating point processor is employed. The purpose of floating point processor is to generate the channel coefficients accurately with high precisions.

\subsection{Simulator design specification}

Figure 1 shows the overall block diagram of the channel simulator. It consists of two DSKs communicating with each other using Multiple Channel Buffered Serial Port 0 (MCBSP0). The TMS320C6713 DSK board acts as a Master device. It generates and transmits channel

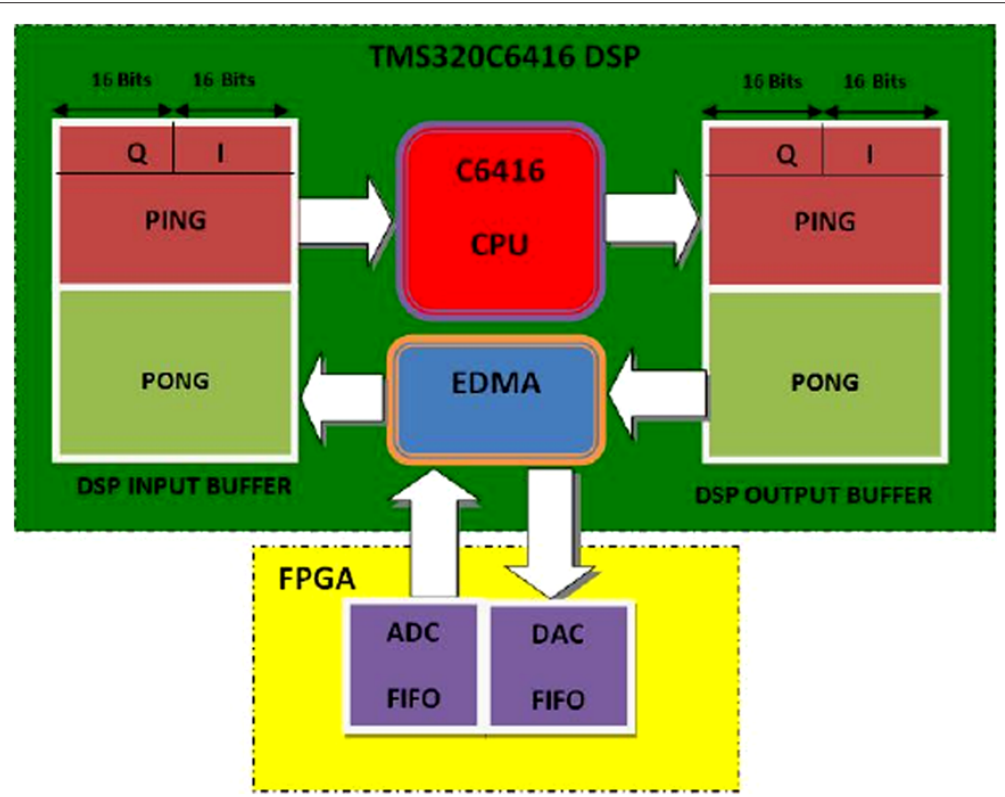

Figure 3 Block diagram showing input output operations. 
Table 1 Timing diagram

\begin{tabular}{|c|c|c|c|c|}
\hline Time Line $\longrightarrow$ & 0 & $N T_{s}$ & $2 N T_{s}$ & $3 N T_{s}$ \\
\hline EDMA Transfer & $\mathrm{ADC} \longrightarrow \mathrm{PING}$ IN & PONG OUT $\longrightarrow$ DAC & $\mathrm{ADC} \longrightarrow \mathrm{PONG}$ IN & PING OUT $\longrightarrow$ DAC \\
\hline DSP Processes & IDLE & PING BUFF & IDLE & PONG BUFF \\
\hline
\end{tabular}

coefficients to the primary TMS320C6416 DSK board which acts as a slave. The system runs according to the following specification:

- TMS320C6416T DSK board having 1 GHz fixed point processor works as a primary board to accept the baseband input and generate output;

- TMS320C6713 DSK board having $225 \mathrm{MHz}$ floating point processor works as a secondary board that will generate channel taps at the required rate;

- Input Baseband data bandwidth $20 \mathrm{MHz}(10 \mathrm{MHz}$ each I \& Q);

- Maximum number of Taps (channel coefficients) generated $\mathrm{N}=8$;

- Maximum Doppler frequency that can be set $=480$ $\mathrm{Hz}$;

- $\quad$ ADC and DAC Buffer size = 1024. Elements $(16 \mathrm{I}+$ 16Q) $=32$ bits;

- $\mathrm{ADC}$ and DAC resolution = 14 bits;

- Maximum sampling rate of $\mathrm{ADC}$ and DAC = $25 \mathrm{MHz}$;

- Transfer rate of channel coefficients $=16 \mathrm{kHz}(2 \mathrm{kHz}$ per tap);

- Maximum excess delay = 1024 samples which on 25 $\mathrm{MHz}$ sampling frequency becomes $41 \mu \mathrm{sec}$.

\subsection{Simulator functionality}

The simulator performs the following tasks.

\subsubsection{Baseband data acquisition}

The baseband data acquisition uses Signalware's ORS-114 daughter board. This card is designed to facilitate rapid construction of prototypes or small to medium production runs with minimum time-to-market. This peripheral card provides flexible analog input and output for applications with a Texas Instruments (TI) Digital Signal Processors (DSP). It mounts on a card that contains TI TMS320C6xxx DSPs made by ORSYS, Inc. These DSP cards, known as the "micro-line" series, contain the processor, DRAM memory and an expansion interface which allows the peripheral card full access to all of the DSP's resources. The hardware block diagram of ORS114 board is shown in Figure 2.

The daughter board is configured to use 2 channels ADC and DAC working at 25 MSPS each and transferring 14 bit data in and out of DSP. The data transfer is done using Enhanced Direct memory Access (EDMA) interface configured with optimal External memory Interface
(EMIF) setting to read and write data. The pin configuration detail is given in [60].

Ping Pong buffering technique described in TI documentations at [61] has been used to perform data transfer efficiently between the I/O devices and Internal Memory (SRAM) of DSP. EDMA engine performs the data transfer between the ping/pong buffers and I/O device alternately and a pingpong flag ensures that the DSP is processing the buffer that is not being overwritten by the EDMA. Since EDMA runs independently from the CPU, the CPU can continue to process the block of data that is in the ping buffer while the EDMA is writing data on the pong buffers and vice versa. In order to remain synchronous with EDMA and void the data loss, it is essential for CPU to finish the processing before the next EDMA interrupt is generated. This Hardware interrupt is generated every time the EDMA completes data transfer.

After reset, the DSP performs all the necessary initializations. It configures EMIF settings, initializes the Daughter board, configures EDMA channels to start data transfer and waits for the peripheral device to input the channel parameters. For the ADC Sampling time $T_{s}$ and PING/PONG buffer size $N$, the data transfer flow is shown in Figure 3. The timing diagram is shown in Table 1.

\subsubsection{Primary secondary board interface}

The function of the primary secondary board interface is to obtain the channel coefficients in real time. For this purpose the Multiple Channel Buffered Serial Port (MCBSP 0) present on the external peripheral interface of the TMS320C6416 DSK board, has been used. The port is directly connected with the MCBSP 0 of the secondary board in Master/Slave configuration such that the secondary board that is generating channel coefficients work

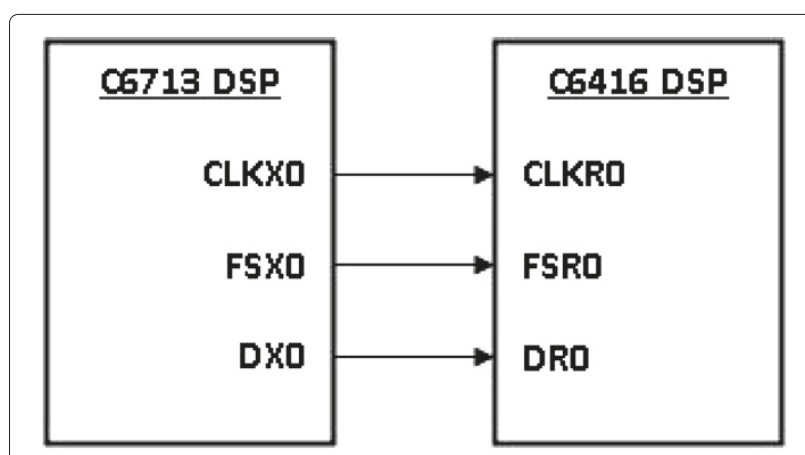

Figure 4 MCBSP connection between the two DSPs. 


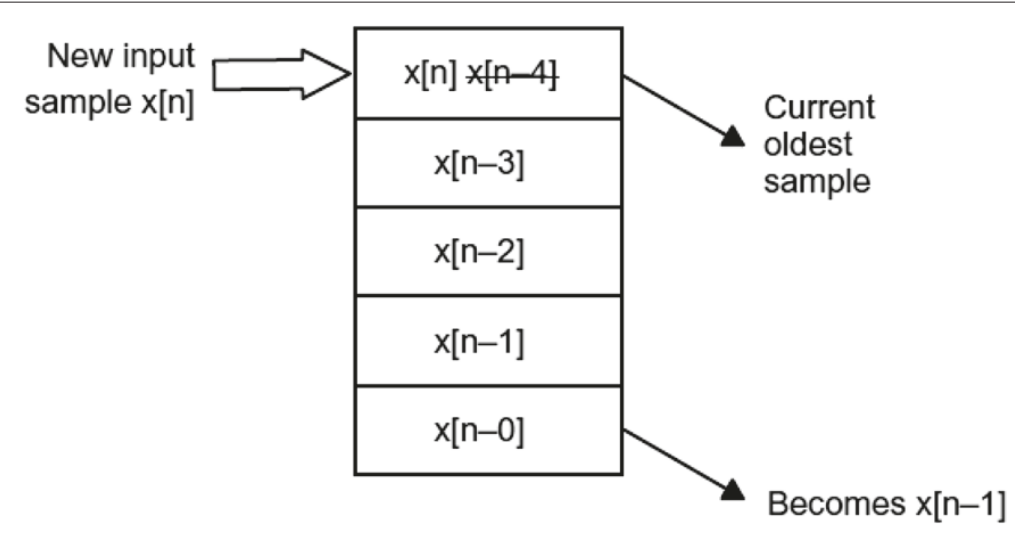

Figure 5 Pointer manipulation using circular addressing.

as Master device as it also generates clock and frame signal for the serial port whereas the primary board acts as a Slave and use these signals to get data. The block diagram of the connection between the two DSPs via MCBSP ports have been shown in the Figure 4.

After connecting the two DSPs together the next step is to configure the ports so that the data can be transmitted and received successfully. The ports are configured by the setting the appropriate values of the four serial port registers. They are Receive Control Register (RCR), Transmit Control Register (XCR), Sample Rate Generator Register (SRGR), Pin Control Register (PCR).

The details of how to set these registers are given in TI documentation [62]. The values are set so that one frame consisting of 8 channel coefficients (each of 32 bits) is transmitted in $500 \mu \mathrm{sec}$ that results in a transmission rate of $16 \mathrm{kHz}$ per coefficient.

Again, EDMA along with ping pong buffering technique is used to perform this transfer efficiently. At the transmitting end, the EDMA interrupt is generated periodically and at the same time, the CPU generates new channel coefficients. Whereas, at the receiving end, when a complete frame is received an interrupt is generated and the channel coefficients are updated.

\subsubsection{Tap delay line filtering}

Tapped Delay Line Filter is the basic block of many digital signal processing applications. It is based on the following equation

$$
y[n]=\sum_{k=0}^{M} x[n-k] . h[k]
$$

where $y[n], x[n], h[n]$ are samples of the output, input and filter coefficient respectively at $n$th sample instant of a digital system of order $M$.

As seen from (6), in order to obtain an output $y[n]$ in real time, a buffer of $M$ previous values (delay line) need to be maintained along with the current sample. Typically, a pointer is set up at the beginning of the sample array (oldest sample) and then manipulated to access the consecutive values.

Whenever a new sample needs to be added to the delay line all the values need to be shifted down. For large values of $M$ (delay line), this will cause additional overhead of shifting the large amount of data. The alternate approach is to overwrite the oldest value. This can be implemented by using circular mode for pointer access.

The input data buffer has finite size and has to be accessed circularly as the new samples are continuously written into the buffer the previously stored (oldest samples) need to be overwritten so that the buffer memory is reused. When the pointer reaches the last location of the buffer, it needs to wrap back to the beginning of the buffer. This would normally involve some amount of software overhead. When Input buffer addressing is defined as circular, the pointer automatically wraps back to the top whenever the bottom of the buffer is reached. Figure 5 illustrates the circular addressing. The input buffer is made circular for that purpose it must be properly aligned in the internal memory. The detail of how to set the buffer as Circular is given in [63].

TDL Filter can be implemented in several ways depending upon the application. Here, the filter is modeled as a frequency selective channel, where the channel taps are

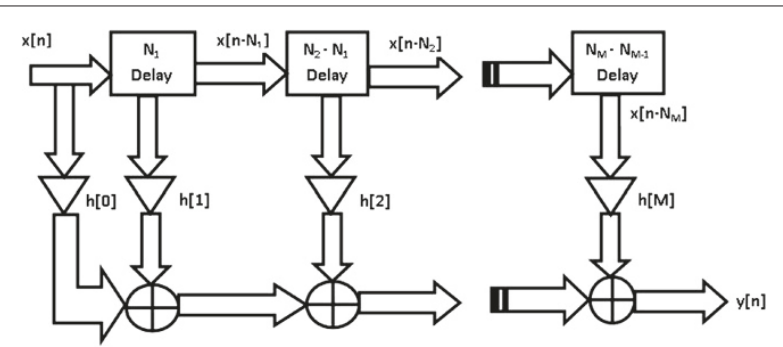

Figure 6 Tap delay line filter model. 
Table 2 Four taps TDL resources allocation for main loop

\begin{tabular}{|c|c|c|c|c|c|c|c|c|}
\hline $\begin{array}{l}\text { FU } \\
\text { Cy }\end{array}$ & 1 & 2 & 3 & 4 & 5 & 6 & 7 & 8 \\
\hline M1 & MPY 14 & MPYH I1 & MPY I1 & MPYH I2 & MPY I2 & MPYH I3 & MPY I3 & MPYH 14 \\
\hline $\mathrm{M} 2$ & MPYHL Q4 & MPYLH Q1 & MPYHL Q1 & MPYLH Q2 & MPYHL Q2 & MPYLH Q3 & MPYHL Q3 & MPYLH Q4 \\
\hline .L1 & SUB I3 & ADD I3 & SUB 14 & ADD 14 & SUB I1 & ADD I1 & SUB 12 & ADD 12 \\
\hline .22 & ADD Q3 & ADD Q3 & ADD Q4 & ADD Q4 & ADD Q1 & ADD Q1 & ADD Q2 & ADD Q2 \\
\hline.$S 1$ & & ADD & $B$ & ZERO & SHR & & $A D D$ & \\
\hline .52 & & & & ZERO & SHR & & & $A D D$ \\
\hline D1 & LDW & LDW & & & LDW & LDW & STH & \\
\hline. $\mathrm{D} 2$ & & & & & SUB & & & STH \\
\hline
\end{tabular}

assumed as multipath fingers located at multiple of sampling time $T_{s}$. The $N=M+1$ tap time intervals are assumed as $\tau_{i}$ where $i$ varies from 0 to $M$ and $\tau_{0}=0$. The channel model is shown in Figure 6. The tap index $N_{i}$ is related with $\tau_{i}$ as

$$
N_{i}=\left\lfloor\frac{\tau_{i}}{T_{s}}+0.5\right\rfloor
$$

where $\lfloor$.$\rfloor indicates the truncation operation. For the$ buffer size $L$, the maximum excess delay that last finger can be computed as

$$
\tau_{\max }=L T_{s}
$$

Using the pipelining approach mentioned in [64] the code has been optimized for $N=8$ taps. The inner loop was completely unrolled to reduce the loop overhead, the dependency graph was created and the instructions were pipelined to reduce the number of cycles. The optimized code consists of 3 parts. The prolog, the mainloop and epilog.
The prolog consists of initialization of local variables, pushing registers over stack for usage inside the function, loading taps coefficients $h[n]$ from memory into registers and defining input buffer as circular. Defining the input as circular buffer removes the overhead of an additional branch instruction inside the loop. The use of circular buffer prevents the constant test of wrapping. The prolog is to be executed once for $L$ size input buffer. It takes 45 cycles to execute this code.

The main loop is also known as kernel of the program which is executed most of the time. It is optimized and instructions are scheduled to maximize the utilization of the CPU resources. For $N$ taps it is executed $2 N$ times per input sample. For 4 taps, the resources allocations are shown in Table 2. It is also shown that loading of data from memory and its storage into memory are done at the same time using .D1 and .D2 functional units. whereas branching instruction, re-initialization, output storing and counter increments have also been scheduled. $S T H$ instructions have been used to store the sample output back into the internal memory, ZERO to re-initialize the output registers back to zero for the computation of

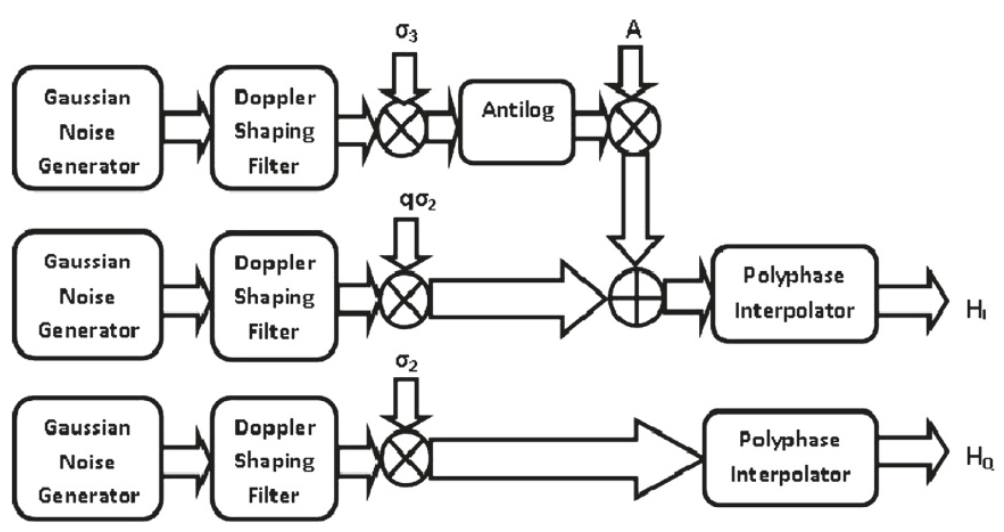

Figure 7 Single tap generation using filter method. 
Table 3 Various channel models for simulation

\begin{tabular}{cccc}
\hline & $\boldsymbol{A}=\mathbf{0}$ & \multicolumn{2}{c}{$\boldsymbol{A} \neq \mathbf{0}$} \\
\cline { 3 - 4 } & & $\boldsymbol{z}(\boldsymbol{t})=\mathbf{0}$ & $\boldsymbol{z}(\boldsymbol{t}) \Rightarrow$ Gaussian \\
\hline$q=1$ & Rayleigh & Rice & Log-Normal Rice \\
\hline $0<q<1$ & Hoyt & Rice Hoyt & Diffused Hoyt \\
\hline$\sigma_{2}=0$ & - & Static & Lognormal \\
\hline
\end{tabular}

next sample output and SHR to bring the output in the required Q3.13 format.

The epilog consists of the remaining part of the function. This include remaining loop portion, popping data back to the registers and branch out of the function. This part takes 42 cycles to execute.

\subsubsection{Channel gains generations}

The channel coefficients have been generated using a floating point TMS320C6713 DSP. Kominakis et al [37] describes the efficient method of generating the channel gains. It uses Infinite Impulse Response (IIR) Doppler filter along with the polyphase interpolator for the generation of correlated Gaussian channel coefficients. The original approach was for flat fading Rayleigh channel only. It was modified for the more generalized 8 taps frequency selective Nakagami-q (Hoyt) mobile to mobile fading channel with diffused LOS.

The block diagram of the channel coefficient generation unit of the single (first) tap is shown in Figure 7. The block diagram represents a generalized channel model. By varying the values of parameters $\left(a, q, \sigma_{2}\right.$ and $\left.A\right)$ different channel models can be obtained and simulated. These models are shown in the Table 3. For $a=0$, the models are obtained for Base to mobile communication whereas $a>0$ represents $\mathrm{V} 2 \mathrm{~V}$ communications.

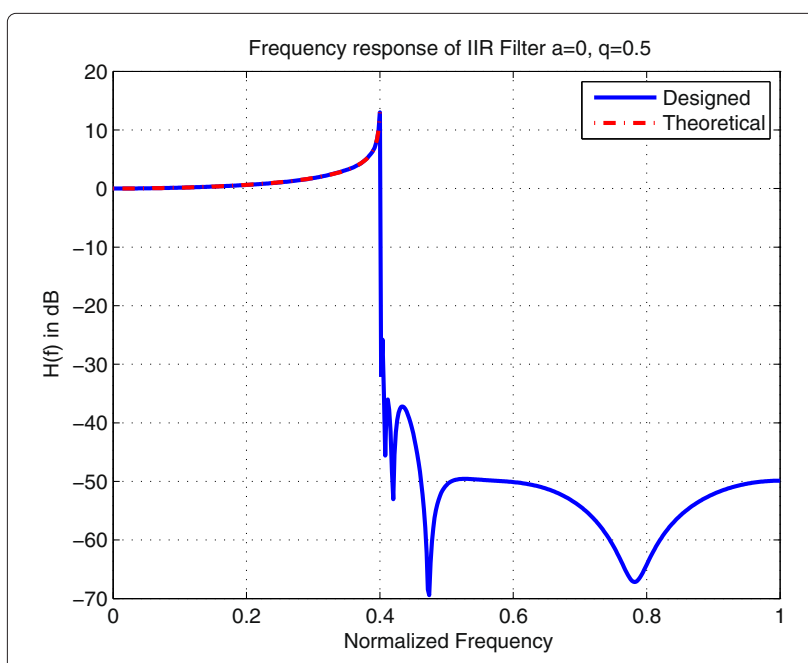

Figure 8 Frequency response of IIR filter $\mathrm{q}=1.0 \mathrm{a}=0$.

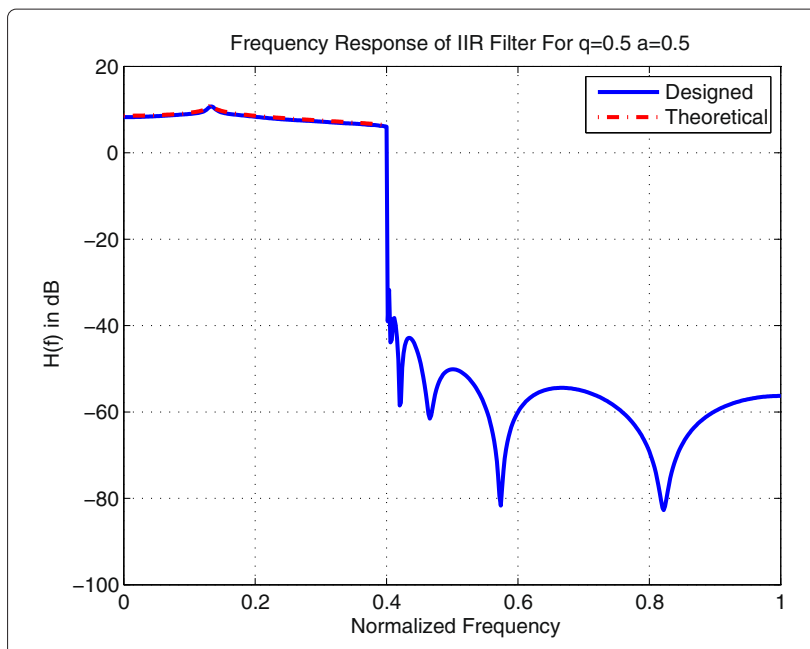

Figure 9 Frequency response of IIR filter $\mathrm{q}=0.5 \mathrm{a}=0.5$.

The Doppler Shaping Filter is implemented as an IIR Filter having the frequency response $\sqrt{S(f)}$ obtained by taking the Square root of (5). The filter has been designed for the Doppler rate of $f_{d} T_{s}=0.2$. The higher rate is achieved by interpolating the channel coefficients $I$ times using polyphase interpolator. For the Fade Rate $\left(f_{d} T_{s}\right)$ of 0.01 , the value of $I=20$ is taken. For the maximum Doppler frequency of $160 \mathrm{~Hz}$ the channel sampling rate is set to $16 \mathrm{kHz}$.

The maximum Doppler frequency is configurable and is set using LCD Keypad interfacing of TMS320C6713 DSK (MCBSP1 port). This can go up to $480 \mathrm{~Hz}$. For a single emulator run, it will remain unchanged. The filter coefficients are computed on the base of normalized Doppler frequency. The algorithm for filter coefficient generation uses fade rate $\left(f_{d} T_{s}\right)$ of 0.01 . For $160 \mathrm{~Hz}$ Doppler the Sampling frequency (Filter coefficient update rate is $16 \mathrm{KHz}$ ).

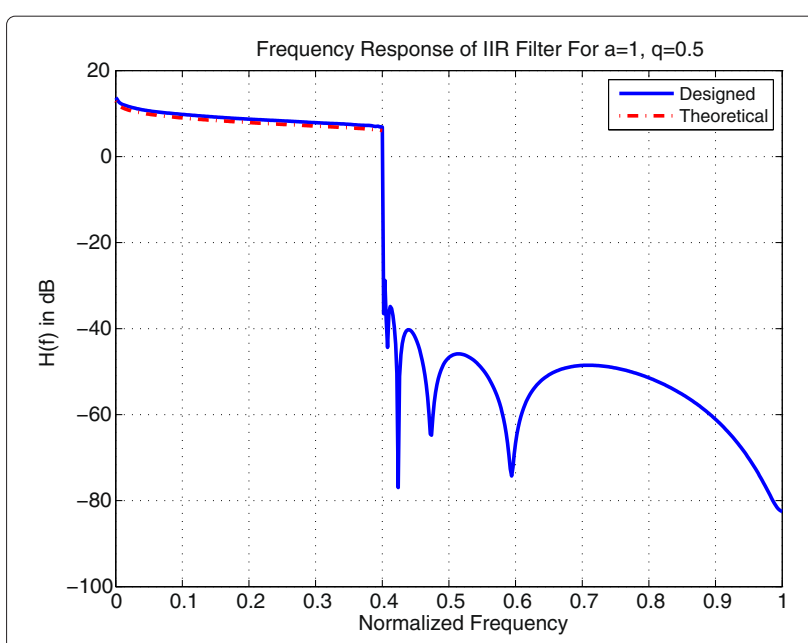

Figure 10 Frequency response of IIR filter $q=0.3 a=1.0$. 


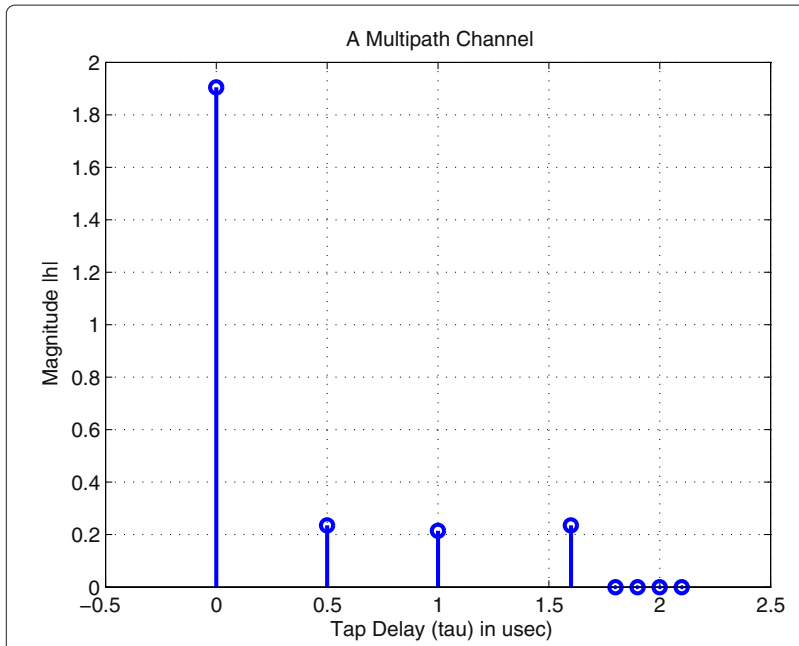

Figure 11 Channel magnitude impulse response.

This means if the Doppler frequency is increased the sampling frequency will also be increased in the same proportion so as to make the fade rate constant. The increase in sampling frequency means MCBSP0 port data rate will be increased. This rate is software configurable and can be set by changing the value of Sample Rate Generator Register (SRGR) of the MCBSP0 port. The upper limit depends upon the complexity of the Channel coefficient generation algorithm and number of taps. For 8 taps, it is $480 \mathrm{~Hz}$ and this can be increased if we further optimize the channel generation code using some optimization techniques (reducing mathematical complexity and efficient use of DSP resources).

The interpolator is implemented as a polyphase filter with a windowed $\operatorname{sinc}($.) function impulse response. The algorithm for channel coefficient generation is modified in order to consider the generalized cases. Figures 8, 9, 10

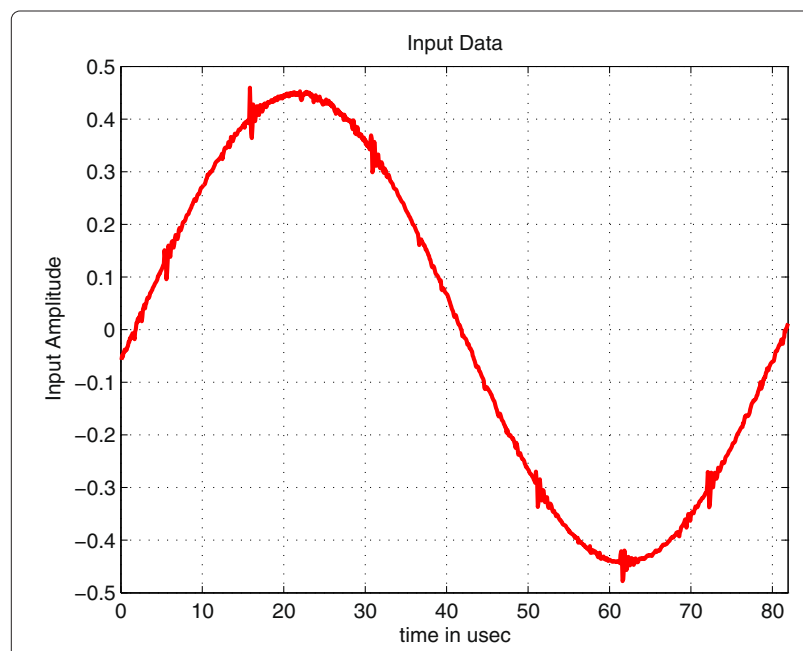

Figure 12 Input data on both I and Q channel.

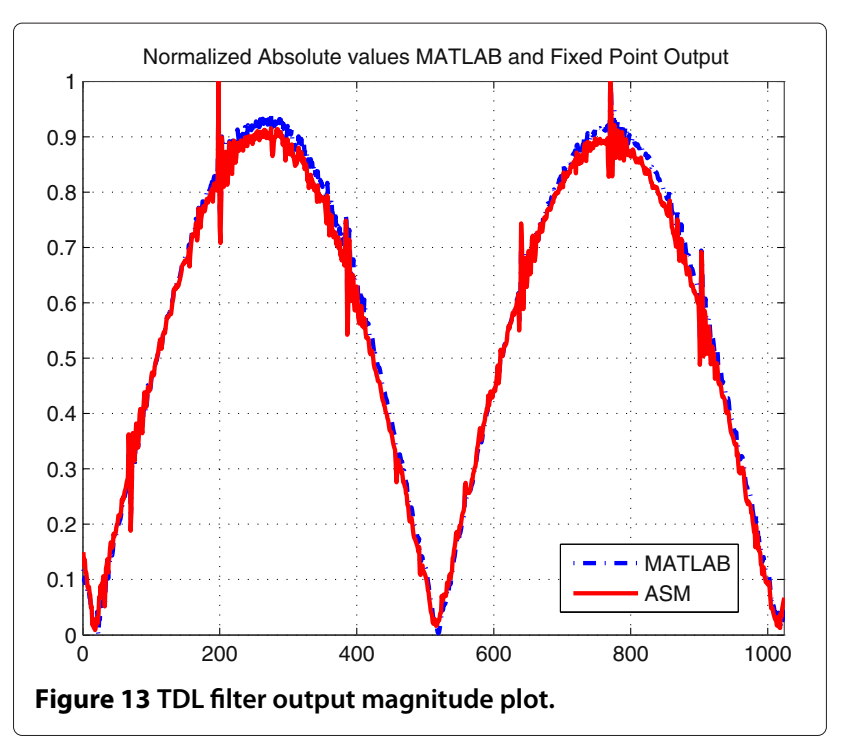

show the frequency response of the modified PSD under different values of $a=(0,0.5,1), q=(1.0,0.5,0.3), A=0$ and $\sigma_{2}>0$.

\section{Results and comparison}

A TDL filter with $\mathrm{N}$ taps is modeled here as a frequency selective multipath channel. Each tap is complex having defined location. The channel is assumed static for one buffer period of time. Both $C$ and assembly functions were executed for $L=1024 \mathrm{I} / \mathrm{O}$ buffer size, data sampling frequency $F s=12.5 \mathrm{MHz}$ and $N=8$ taps filter coefficients having values $\mathrm{h}=\{1.8362-0.5073 \mathrm{i},-0.2169+0.0915 \mathrm{i}$, $0.1448+0.1585 \mathrm{i},-0.2169+0.0915 \mathrm{i}, 0.0,0.0,0.0,0.0\}$ with corresponding tap locations at $\{0,0.5,1,1.6,1.8,1.9,2.0$, $2.1\}$ usec. The magnitude response $|h|$ of the channel is shown in Figure 11.

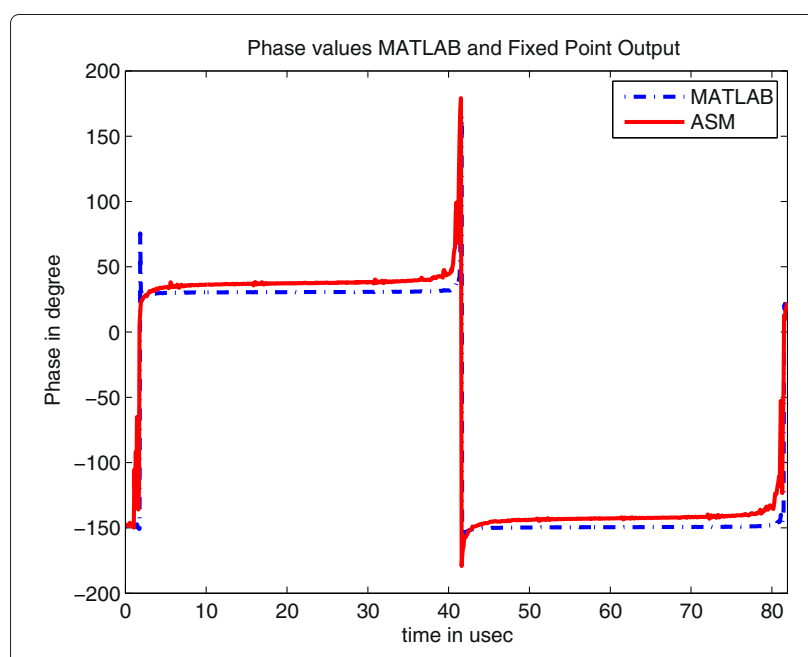

Figure 14 TDL filter output phase plot. 


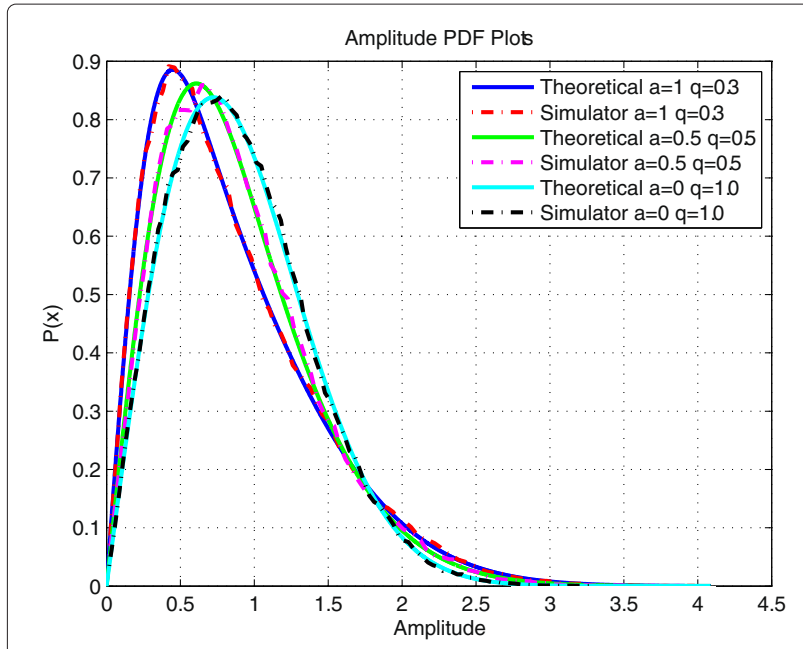

Figure 15 PDF plot for amplitude.

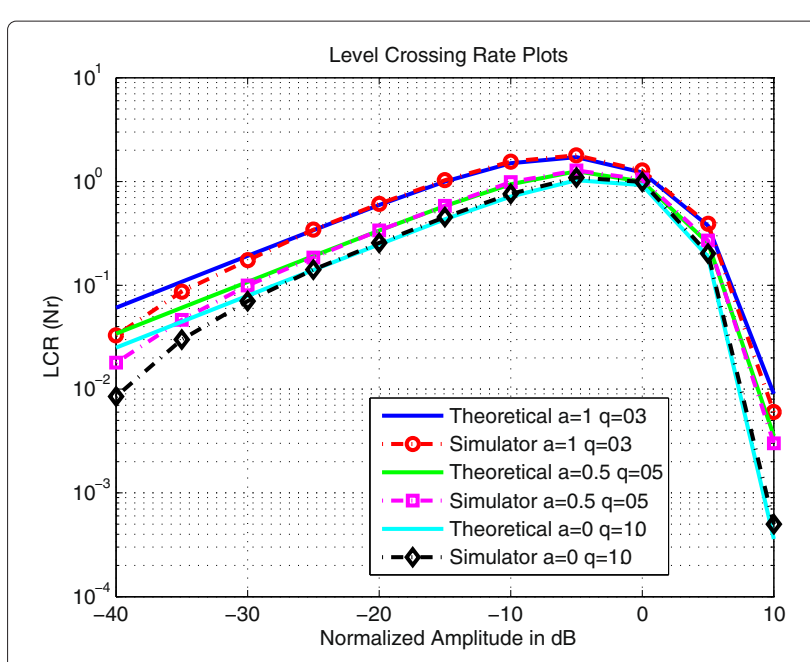

Figure 17 PDF plot for LCR.
Most V2V systems operate in frequency range 5-5.9 $\mathrm{GHz}$ [57]. Bwang et el [65] and Matolak et el [66] used 5.15 and $5.2 \mathrm{GHz}$ carrier frequencies to simulate the V2V channel. Hence, the simulation was run with the following parameters, carrier frequency $f_{c}=5.8 \mathrm{GHz}$, velocity of receiver $V_{1}=30 \mathrm{~km} / \mathrm{hr}$ which means $f_{m 1}=160 \mathrm{~Hz}$, LOS component $A=0$ for Hoyt model, three different values of $q=1,0.5,0.3$ and three different values of $a=0,0.5,1$.

The sinusoidal input shown in Figure 12 is applied on both I \& Q channels. The output of both were found exactly match with each other. For fixed point $C$ code, it takes 1460 cycles per sample to generate output whereas the optimized code gives output in 16 cycles per sample.
From (8), the maximum excess delay $\tau_{\max }$ the system can have is found to be 81.92 usec.

A comparison has been made for a given complex input between the outputs of the MATLAB complex FIR filter code with the fixed point assembly code and are shown in Figure 13 (Magnitude plot) and Figure 14 (Phase plot). The number of taps assumed are $N=8$ with buffer size $L=1024$. For C6416 DSP operating at $1 \mathrm{GHz}$, the cycle time becomes 1 ns hence proposed algorithm will take around $16 n s$ per sample which means that data with around $60 \mathrm{MHz}$ sampling frequency can be processed.

In order to verify the channel coefficient generation, the performance analysis of the Channel has also been

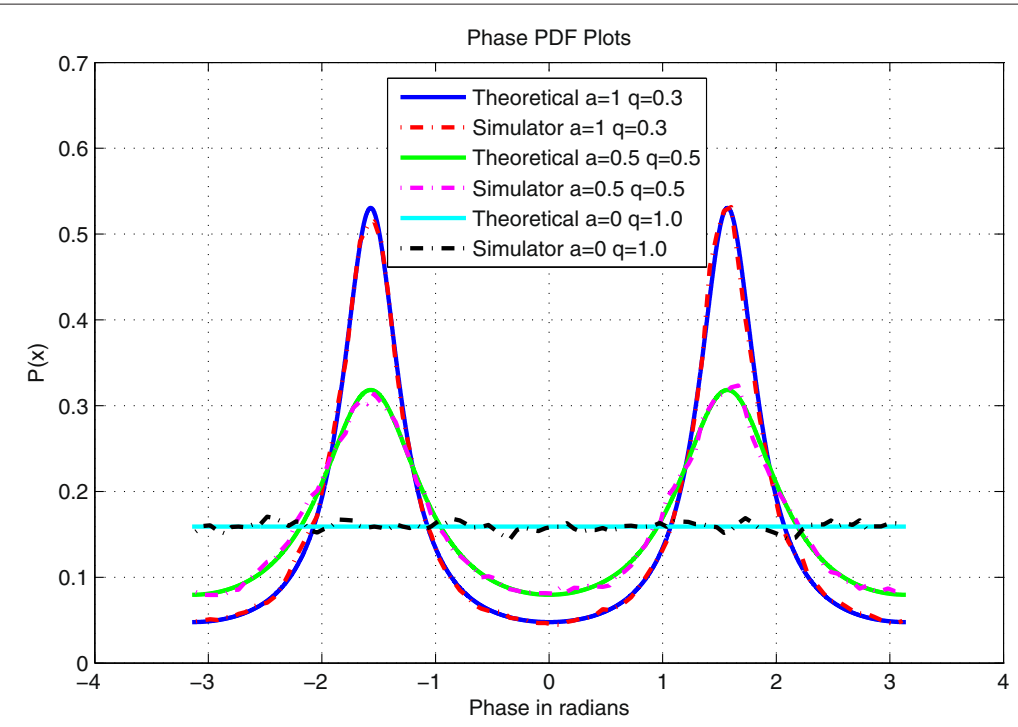

Figure 16 PDF plot for phase. 


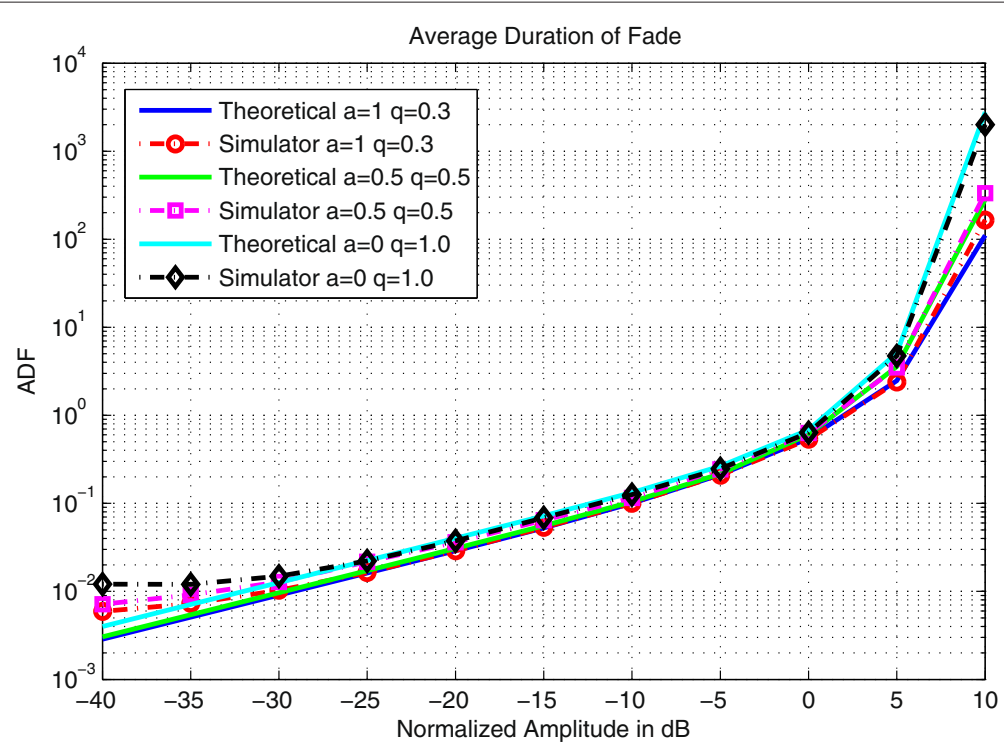

Figure 18 PDF plot for ADF.

done. The BPSK modulated data is applied at the input of the single tap channel and the output and Channel coefficients (400k samples) are stored in the SDRAM of TMS320C6416 DSP Board in real time. Since performance analysis is independent of data rate and sampling frequency, hence due to the limited size of SDRAM the sampling rate was set to $2 \mathrm{MHz}$ and the input data rate to $200 \mathrm{kbps}$. The amplitude and phase Probability Density Function (PDF), Level Crossing rate (LCR), Average
Duration of Fade (ADF) and Bit Error Rate (BER) plots are shown in the Figure 15, 16, 17, 18, 19 respectively. The plots are found to be closely matched with the corresponding theoretical plots. Mean square error (MSE) between the theoretical and simulated values of the amplitude, phase PDF, BER curves and LCR are shown in Table 4.

The proposed simulator is also compared with the one described in [67]. There is a significant difference between

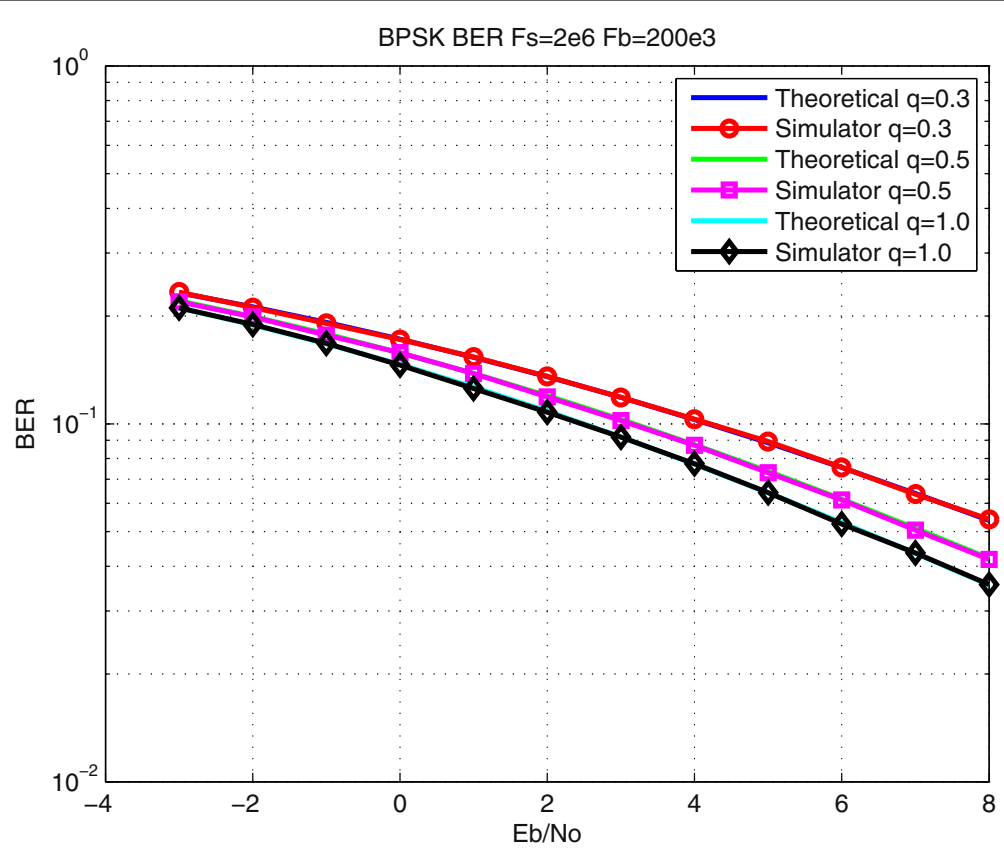

Figure 19 BER plot, BPSK modulation $\mathrm{q}=1, \mathrm{q}=0.5, \mathrm{q}=0.3$. 
Table 4 MSE of various quantities for $a=0.5$

\begin{tabular}{ccccc}
\hline $\boldsymbol{q}$ & Envelope PDF & Phase PDF & BPSK BER & LCR \\
\hline 0.3 & $4.240 \times 10^{-5}$ & $4.647 \times 10^{-5}$ & $1.456 \times 10^{-7}$ & $13 \times 10^{-4}$ \\
\hline 0.5 & $8.148 \times 10^{-5}$ & $3.371 \times 10^{-5}$ & $4.439 \times 10^{-7}$ & $6 \times 10^{-4}$ \\
\hline 1.0 & $6.511 \times 10^{-5}$ & $2.586 \times 10^{-5}$ & $0.775 \times 10^{-7}$ & $11 \times 10^{-4}$
\end{tabular}

the philosophies of the two simulators. The simulator described in [67] requiring measured impulse response, generates channel coefficients from the measured channel transfer function. These channel coefficients operate on the information data to deliver performance in terms of error rate. This simulator essentially requires in field measurements. The proposed simulator does not require measured in-field data but uses the statistical models available in published standards, thus saving significantly on the cost of expensive field trials. The proposed simulator operates on the statistical parameters to generate real time channel coefficients, which then operate on the information data to generate performance in terms of error rates. The developed simulator can be used to operate on the in-field channel data provided we augment this simulator with a facility to convert the stored channel data into channel coefficients or channel statistics. It should be borne in mind that our interest has been to replace field environment by laboratory environment and by various options on choices of channel character.

\section{Conclusion}

In this paper, design and implementation of an efficient real time wideband simulator has been discussed. The simulator was run in real time with a known input and the output data was analyzed. The TDL filter has been optimally implemented over TMS320C6416 DSP. The output of the filter has been verified by comparing the simulator output with MATLAB. The pipelined architecture of the processor and the circular buffer have been efficiently utilized. The channel coefficients have been generated and analyzed. The BPSK modulated data has been input and the output has been stored. The bit error rate has been measured and compared with the theoretical data to verify the validity of the channel simulator.

\section{Competing interests}

The authors declare that they have no competing interests.

\section{Authors' contributions}

DSP based wideband real time simulator has been developed that is based on Nakagami Hoyt V2V channel model and is flexible to simulate the other channel models by just changing the parameters. Both authors read and approved the final manuscript.

\section{Acknowledgements}

The authors would like to acknowledge the support provided by King Abdulaziz City for Science and Technology (KACST) through the Science and Technology Unit at King Fahd University of Petroleum and Minerals (KFUPM) for funding this work through project No. NSTP08-ELEC42-4 as part of the National Science, Technology and Innovation Plan (NSTIP).

Received: 1 May 2012 Accepted: 25 October 2012

Published: 1 December 2012

\section{References}

1. K Bullington, Radio propagation variations at VHF and UHF. Proc. IRE. 32, 27-32 (1950)

2. JJ Egli, Radio propagation above $40 \mathrm{MHz}$ over irregular terrain. Proc. IRE 45(10), 1383-1391 (1957)

3. WC Jakes, DO Reudink, Comparison of mobile radio transmission at UHF and X band. Vehicular Technol. IEEE Trans. 16, 10-14 (1967)

4. PM Trifonov, VN Buelko, VS Zotov, Structure of USW field strength spatial fluctuations in a city. Trans. Telecomm. Radio Eng. 9, 26-30 (1964)

5. Y Okamura, YA Kol, Field strength and its variability in VHF and UHF land-mobile radio service. Rev. Elec. Comm. Lab. 9-10, 825-873 (1968)

6. GL Turin, FD Clapp, TL Johnston, SB Fine, D Lavry, A statistical model of urban multipath propagation. IEEE Trans. Veh Technol. 21, 19 (1972)

7. DM Black, DO Reudink, Some characteristics of mobile radio propagation at $836 \mathrm{MHz}$ in the Philadelphia area. Vehicular Technol. IEEE Trans. 21, 45-51 (1972)

8. D Cox, Delay Doppler characteristics of multipath propagation at 910 $\mathrm{MHz}$ in a suburban mobile radio environment. Antennas Propagation. IEEE Trans. 20, 625-635 (1972)

9. D Cox, Time- and frequency-domain characterizations of multipath propagation at $910 \mathrm{MHz}$ in a suburban mobile-radio environment. Radio Sci. 7, 1069-1077 (1972)

10. TAulin, Characteristics of a digital mobile radio channel. Rev. Elec. Comm. Lab. 30, 45-53 (1981)

11. SY Seidel, TS Rappaport, $914 \mathrm{MHz}$ path loss prediction models for wireless communications in multifloored buildings. Rev. Elec. Comm. Lab. 40, 207-217 (1992)

12. DA Hawbaker, TS Rappaport, Indoor wideband radiowave propagation measurements at 1.3 GHz and 4.0 GHz. IEE Electron. Lett. 26, 1800-1802 (1990)

13. U Dersch, J Troger, E Zollinger, Multiple reflections of radio waves in a corridor. IEEE Trans Antenna Propagation. 42, 1571-1574 (1994)

14. F Babich, G Lombardi, Statistical analysis and characterization of the indoor propagation channel. Rev Elec Comm Lab. 48, 455-464 (2000)

15. X Zhao, J Kivinen, P Vainikainen, K Skog, Propagation characteristics for wideband outdoor mobile communications at $5.3 \mathrm{GHz}$. IEEE J Selected Areas Commun. 20, 507-514 (2002)

16. N Youssef, CX Wang, M Patzold, A study on the second order statistics of Nakagami-Hoyt mobile fading channels. IEEE Trans Vehicular Technol. 54, 1259-1265 (2005)

17. SO Rice, Statistical properties of a sine wave plus random noise. Bell Syst J. 27, 109-157 (1948)

18. RS Hoyt, Probability functions for the modulus and angle of the normal complex variate. Bell Syst J. 26, 318-359 (1947)

19. M Nakagami, The m-distribution - A General Formula of Intensity Distribution of Rapid Fading. (Permagon Press, Oxford, 1960)

20. MA Taneda, J Takada, K Araki, in IEEE International Symposium on Personal, Indoor, and Mobile Radio Communications. A new approach to fading (Weibull model, 1999), pp. 711-715

21. F Hansen, FI Meno, Mobile fading-Rayleigh and lognormal superimposed. IEEE Trans. Veh Technol. 36, 332-335 (1977)

22. R Mangharam, J Meyers, R Rajkumar, Stancil D, in Proceedings of Society for Automotive Engineers (SAE) World Congress. A multi-hop mobile networking test-bed for telematics (Detroit, USA, 2005)

23. J Dulmage, M Tsai, M Fitz, Daneshrad B, in Proceedings of the 1st international workshop on Wireless network testbeds, experimental evaluation \& characterization. COTS-based DSRC testbed for rapid algorithm development, implementation, and test (WINTECH '06, New York, USA, 2006)

24. TM Fernandez, JA Garcya, ML Gonzalez, L Castedo, in Proceedings of 8th International Conference on ITS Telecommunications, 2008. ITST 2008. FlexVehd: a flexible test bed for vehicular radio interfaces (Hilton Phuket, Thailand, 2008), pp. 283-287

25. AA Gaston, WH Chriss, A Multipath fading simulator for mobile radio. IEEE Trans. Vehicular Technol. 22, 241-244 (1973) 
26. RC Fitting, Widebandtroposcatterradiochannelsimulator Trans. IEEE Commun. Technol. 15, 565-570 (1975)

27. JR Ball, A real-time fading simulator for mobile radio. Radio Electron. Eng $\mathbf{5 2}, 475-478(1982)$

28. M Lecours, F Marceau, Design and implementation of channel simulator for wideband mobile radio transmission. In Proc. Vehicular Technol. Conf. (VTC). 2, 652-655 (1989)

29. RA Comroe, F Marceau, All-digital fading simulator. In Proc. Nut. Electron Conf. 32, 136-139 (1978)

30. RA Goubran, HM Hafez, AUH Sheikh, Real-time programmable land mobile channel simulator. Vehicular Technol. Conf., 1986 36th IEEE. 36, 215-218 (1986)

31. JF An, AMD Turkmani, JD Parson, Implementation of a DSP-based frequency non-selective fading simulator. Fifth Int. Conf. Radio Receivers Assoc. Syst, 20-24 (1990)

32. PJ Cullen, PC Fannin, A Garvey, Real-time simulation of randomly time-variant linear systems: the mobile radio channel. IEEE Trans. Instrum. Meas. 43, 583-591 (1994)

33. XF Chen, KS Chung, Generation of noise sources for a digital frequency selective fading simulator. Int. Symp. Signal Process. Appl., ISSPA. 2, 463-466 (1996)

34. AK Salkintzis, Implementation of a digital wide-band mobile channel simulator. Broadcasting. IEEE Trans. 45, 122-128 (1999)

35. JR Papenfuss, MA Wickert, Implementation of a real-time, frequency selective, RF channel simulator using a hybrid DSP-FPGA architecture. IEEE Radio Wireless Conf., 2000. 49, 135-138 (2000)

36. S Fischer, R Seeger, KD Kammeyer, Implementation of a real-time satellite channel simulator for laboratory and teaching purposes. The Third European DSP Education \& Research Conference (2000). [http://www.ti. com/europe/docs/univ/docs/info.htm]

37. C Komninakis, A Fast, Telecommunications, accurate rayleigh fading simulator. Global Conf., 2003. GLOBECOM '03. IEEE. 6, 3306-3310 (2003)

38. M Khars, C Zimmer, Digital signal processing in a real time propagation simulator. IEEE Trans. Instrum Meas. 55, 197-205 (2006)

39. S Kandeepan, ADS Jayalath, in Proceedings of International Conference on Communication Systems, 2006. ICCS 2006. 10th IEEE Singapore. Narrow-band channel simulator based on statistical models implemented on Texas instruments C6713 DSP and national instruments PCIE-6259 hardware, (Singapore, 2006), pp. 1-6

40. A Ghazel, E Boutillon, JL Danger, G Gulak, H Laamari, Design and performance analysis of a high speed AWGN communication channel emulator. IEEE Pac. Rim Conf. Commun., Comput. Signal Process. 1, 374-375 (2001)

41. F Sattar, M Mufti, DD Stancil, P Steenkiste, in Proceedings of the 16th International Conference on Microelectronics, 2004. ICM 2004. VLSI architecture of Rayleigh fading simulator based on IIR filter and polyphase interpolator, (Tunisia, 2004), pp. 291-294

42. S Picol, G Zaharia, GE Zein, Towards the development of a hardware simulator for MIMO radio channels. ignals. Circuits Syst. 2005. ISSCS 2005. Int. Symp. 2005, 1, 115-118 (2005)

43. S Picol, G Zaharia, Houzet, GE Zein, in Proceedings of IEEE 68th Vehicular Technology Conference, 2008. VTC 2008-Fall. Hardware simulator for MIMO Radio channels: design and features of the digital block, (Alberta, 2008), pp. 1-5

44. F Carames, M Gonzalez-Lopez, L Castedo, in 9th International Conference on Intelligent Transport Systems Telecommunications, (ITST). FPGA-based vehicular channel emulator for evaluation of IEEE $802.11 \mathrm{p}$ transceivers, (Lille, France, 2009), pp. 592-597

45. KC Borries, G Judd, DD Stancil, P Steenkiste, FPGA-Based channel simulator for a wireless network emulator. IEEE Vehicular Technol Conf, 1-5 (2009)

46. AS Akki, F Haber, A statistical model for mobile-to-mobile land communication channel. IEEE Trans. Veh. Technol. 35, 2-7 (1986)

47. AS Akki, Statistical properties of mobile-to-mobile land communication channels. IEEE Veh. Technol. Conf. 43, 826-831 (1994)

48. DW Matolak, I Sen, X Wenhui, NT Yaskoff, $5 \mathrm{GHZ}$ wireless channel characterization for vehicle to vehicle communications. Mil. Commun. Conf. 5, 3016-3022 (2005)

49. R Wang, D Cox, Channel modeling for adhoc mobile wireless networks. Proc IEEE Veh. Technol. Conf. 1, 21-25 (2002)
50. CS Patel, GL Stuber, TG Pratt, Simulation of Rayleigh faded mobile-to-mobile comimunication channels. Vehicular Technol. Conf 2003. VTC 2003-Fall. 2003 IEEE 58th. 1, 163-167 (2003)

51. A Petrolino, J Gomes, G Tavares, in IEEE Vehicular Technology Conference, 2008. A Mobile-to-Mobile Fading Channel Simulator Based on an Orthogonal Expansion (VTC Spring 2008, Marina Bay, Singapore, 2008), pp. $366-370$

52. KC Borries, DD Stancil, in IEEE 65th, Vehicular Technology Conference, 2007. VTC2007-Spring. Effcient Simulation of Mobile-To-Mobile Rayleigh Fading using Gaussian Quadrature, (Dublin, Ireland, 2006), pp. 534-538

53. AG Zaji, GL Stuber, A new simulation model for mobile-to-mobile Rayleigh fading channels. Wireless Commun. Networking Conf., 2006. WCNC 2006. IEEE. 3, 1266-1277 (2006)

54. Ml Akram, AU Sheikh, in Wireless Communications and Networking Conference Workshops (WCNCW), 2012 IEEE. Modeling Nakagami Hoyt mobile to mobile fading channel with diffused line of sight, (Paris, France, 2012), pp. 398-403

55. AS Akki, Modeling and characterization of land mobile communication. PhD thesis. (Uniersrsity of Pennsilvenia, Department of Electrical Engineering, Philadelphia, Pennsylvania, United States, 1977)

56. CF Mecklenbrauker, AF Molisch, J Karedal, F Tufvesson, A Paier, L Bernado, T Zemen, O Klemp, N Czink, Vehicular channel characterization and its implications for wireless system design and performance. Proc. IEEE. 99, 1189-1212 (2011)

57. A Molisch, F Tufvesson, J Karedal, C Mecklenbrauker, A survey on vehicle-to-vehicle propagation channels. Wireless Commun., IEEE. 16, 12-22 (2009)

58. AK Papazafeiropoulos, SA Kotsopoulos, An extended generalized rice model for wireless communications. Vehicular Technol. IEEE Trans. 59, 2604-2609 (2010)

59. DW Matolak, I Sen, W Xiong, in Mobile and Ubiquitous Systems Workshops, 2006. 3rd Annual International Conference. Channel modeling for V2V communications, (San Jose, California, 2006), pp. 1-7

60. Documentation package of ORS-114 Wide bandwidth Microline Analog peripheral card. Version 1.02005

61. Texas Instrument web site. [http:\www.ti.com]

62. SPRA455A, Using the TMS320C6000 MCBSP as a High Speed Communication Port. Texas Instruments (2001)

63. SPRA645A, Circular Buffering on TMS320C6000. Texas Instruments (2001)

64. SPRU198G, TMS320C6000 Programmer's Guide. Texas Instruments (2002)

65. B Wang, I Sen, DW Matolak, in IEEE 66th, Baltimore, Vehicular Technology Conference, VTC-2007 Fall. Performance evaluation of 802.16e in vehicle to vehicle channels, MD, USA, 2007), pp. 1406-1410

66. I Sen, DW Matolak, V2V Channels and performance of multi-user spread spectrum modulation. Proc. IEEE 1st Intx'l. Symp, Wireless Vehic. Commun. 2, 19-25 (2007)

67. K Khokhar, S Salous, Frequency domain simulator for mobile radio channels and for IEEE 802.16-2004 standard using measured channels. Commun, IET. 2, 869-877 (2008)

doi:10.1186/1687-1499-2012-359

Cite this article as: Akram and Sheikh: Design and implementation of real time wideband channel simulator. EURASIP Journal on Wireless Communications and Networking 2012 2012:359. 